Real Analysis Exchange

Vol. 24(1), 1998/99, pp. 447-456

Ian June L. Garces, Department of Mathematics, Ateneo de Manila University, P.O. Box 154, 1099 Manila, The Philippines

Lee Peng-Yee, Division of Mathematics, National Institute of Education, Singapore 259756, e-mail: leepy@@am.nie.ac.sg

Zhao Dongsheng, Division of Mathematics, National Institute of Education, Singapore 259756, e-mail: leepy@@am.nie.ac.sg

\title{
MOORE-SMITH LIMITS AND THE HENSTOCK INTEGRAL
}

\begin{abstract}
An integral is defined using the Moore-Smith limit and this new integral is compared to the Henstock integral.
\end{abstract}

It is well-known though not easily found in the literature that the Riemann integral can be defined by Moore-Smith limit using divisions. Then many properties of the Riemann integral will have straightforward proofs. In this paper, we shall investigate whether the Henstock integral can also be defined by means of Moore-Smith limit involving $\delta$-fine divisions. We assume that the reader is familiar with the definition of the Henstock integral [3].

A division $D$ of $[a, b]$ is a finite set of interval-point pairs $([u, v], \xi)$ such that the intervals $[u, v]$ from $D$ are non-overlapping and their union is $[a, b]$ and also $\xi \in[u, v]$ for each $([u, v], \xi) \in D$. Let $D_{1}=\{([u, v], \xi)\}$ and $D_{2}=\{([s, t], \eta)\}$ be two divisions of $[a, b]$. Then $D_{2}$ is said to be finer than $D_{1}$ in the Riemann sense, or in symbols, $D_{2} \sqsupseteq D_{1}$ if for each $([s, t], \eta) \in D_{2}$ we have $[s, t] \subset[u, v]$ for some $([u, v], \xi) \in D_{1}$ and when $[s, t]=[u, v]$ we have $\eta=\xi$. Then $(\mathcal{D}, \sqsupseteq)$ is a directed set of divisions $D$ of $[a, b]$. More precisely, the following conditions are satisfied:

1. $D \sqsupseteq D$ for all $D \in \mathcal{D}$;

2. if $D_{1}, D_{2}, D_{3} \in \mathcal{D}$ with $D_{1} \sqsupseteq D_{2}$ and $D_{2} \sqsupseteq D_{3}$, then $D_{1} \sqsupseteq D_{3}$;

3. if $D_{1}, D_{2} \in \mathcal{D}$ with $D_{1} \sqsupseteq D_{2}$ and $D_{2} \sqsupseteq D_{1}$, then $D_{1}=D_{2}$; and

Mathematical Reviews subject classification: Primary: 26A39

Received by the editors November 27, 1997 
4. for every $D_{1}, D_{2} \in \mathcal{D}$, there exists $D_{3} \in \mathcal{D}$ such that $D_{3} \sqsupseteq D_{1}$ and $D_{3} \sqsupseteq D_{2}$.

Hence the Riemann integral of $f$ on $[a, b]$ is the Moore-Smith limit [1] of Riemann sums using $(\mathcal{D}, \sqsupseteq)$. In symbols,

$$
\int_{a}^{b} f=\lim _{D \in \mathcal{D}}(D) \sum f(\xi)(v-u)
$$

We recall that a function $f$ is Henstock integrable to $A$ on $[a, b]$ if for every $\epsilon>0$ there exists $\delta(x)>0$ such that for any $\delta$-fine division $D$ of $[a, b]$ we have

$$
\left|(D) \sum f(\xi)(v-u)-A\right|<\epsilon
$$

A division $D$ of $[a, b]$ is $\delta$-fine if $\xi \in[u, v] \subset(\xi-\delta(\xi), \xi+\delta(\xi))$ for each $([u, v], \xi) \in D$. Now let $\mathcal{D}$ be the family of $\delta$-fine divisions of $[a, b]$ for some given $\delta(x)>0$. For $D_{1}, D_{2} \in \mathcal{D}$, we write $D_{2} \geq D_{1}$ and say that $D_{2}$ is finer than $D_{1}$ in the Henstock sense using $\delta$ if for every $([s, t], \eta) \in D_{2}$ we have $[s, t] \subset[u, v]$ for some $([u, v], \xi) \in D_{1}$, and $\left\{\xi:([u, v], \xi) \in D_{1}\right\} \subset\{\eta$ : $\left.([s, t], \eta) \in D_{2}\right\}$. Then $(\mathcal{D}, \geq)$ is a directed set. A function $f$ is said to be $H_{1}$-integrable to $A$ on $[a, b]$ if $A$ is the Moore-Smith limit of the Riemann sums using the directed set $(\mathcal{D}, \geq)$. More precisely, there exists $\delta(x)>0$ such that for every $\epsilon>0$ there exists a $\delta$-fine division $D_{0}$ such that for every $\delta$-fine division $D \geq D_{0}$ we have

$$
\left|(D) \sum f(\xi)(v-u)-A\right|<\epsilon .
$$

We say that $A$ is the $H_{1}$-integral of $f$ on $[a, b]$ and that $f$ is $H_{1}$-integrable on $[a, b]$ using $\delta$. Note the difference that here we choose $\delta(x)$ first then $\epsilon$, whereas in the definition of the Henstock integral $\delta(x)$ comes after $\epsilon$.

Example 1. A Riemann integrable function on $[a, b]$ is $H_{1}$-integrable there using an arbitrary $\delta(x)>0$, and the two integrals are equal.

Example 2. The Dirichlet function, given by $f(x)=1$ when $x$ is rational and 0 when $x$ is irrational, is $H_{1}$-integrable on $[0,1]$, using $\delta$ where $\delta\left(r_{i}\right)=2^{-i}$ for $i=1,2, \ldots$ and $\left\{r_{1}, r_{2}, \ldots\right\}$ are the rational numbers in $[0,1]$.

Example 3. Let $f(x)=F^{\prime}(x)$ where $F(0)=0$ and $F(x)=x^{2} \sin x^{-2}$ where $x \neq 0$. Then $f$ is $H_{1}$-integrable on $[0,1]$, using $\delta$ where $\delta(x)=\delta_{n}$ when $x \in(1 /(n+1), 1 / n]$ for some suitable $\delta_{n}$ and $n=1,2, \ldots$, and arbitrary when $x=0$. 
It is easy to see that every $H_{1}$-integrable function on $[a, b]$ is also Henstock integrable there. We will be using this fact frequently in the succeeding discussion.

For convenience, we say that $f$ is $H_{1}$-integrable on a set $X \subset[a, b]$ if $f \mathcal{X}_{X}$ is $H_{1}$-integrable on $[a, b]$ where $\mathcal{X}_{X}$ denotes the characteristic function of $X$. We may define the primitive $F$ of $f$ on $[a, b]$ with $f(x)=0$ for $x \in[a, b]-X$. It is easy to see that if $f$ is $H_{1}$-integrable on $X_{1}$ using $\delta_{1}$ and on $X_{2}$ using $\delta_{2}$ then $f$ is $H_{1}$-integrable on the union $X_{1} \cup X_{2}$ using $\delta=\min \left\{\delta_{1}, \delta_{2}\right\}$. However, we have the following.

Lemma 4. Let $f$ be $H_{1}$-integrable on a closed set $X_{1} \subset[a, b]$ using $\delta_{1}$, and on another closed set $X_{2} \subset[a, b]$, with $f(x)=0$ for $x \notin X_{1} \cup X_{2}$. If the primitive $F$ of $f$ on $[a, b]$ is absolutely continuous there, then $f$ is $H_{1}$-integrable on $X_{1} \cup X_{2}$ using $\delta$, where $\delta(x)=\delta_{1}(x)$ when $x \in X_{1}$.

Proof. We may assume $X_{1} \subset X_{2}$. Suppose $f$ is $H_{1}$-integrable on $X_{2}$ using $\delta_{2}$. Then for every $\epsilon>0$ there exists a $\delta_{i}$-fine division $D_{i}$ on $[a, b], i=1,2$, such that for any $\delta_{i}$-fine division $D \geq D_{i}$ we have

$$
\left|(D) \sum_{\xi \in X_{i}} f(\xi)(v-u)-A_{i}\right|<\epsilon,
$$

where $A_{i}$ denotes the $H_{1}$-integral of $f$ on $X_{i}$. We may assume $\delta_{2}(x) \leq \delta_{1}(x)$ for all $x \in[a, b]$. Put $\delta(x)=\delta_{1}(x)$ when $x \in X_{1}$ and $\delta_{2}(x)$ when $x \in[a, b]-X_{1}$. We may modify $\delta_{2}(x)$, if necessary, so that $\left(x-\delta_{2}(x), x+\delta_{2}(x)\right) \cap X_{1}=\emptyset$ when $x \notin X_{1}$.

Since $F$ is absolutely continuous on $[a, b]$, there exists $\eta>0$ such that for any partial division $D$ of $[a, b]$ we have

$$
\left|(D) \sum F(u, v)\right|<\epsilon \quad \text { whenever } \quad(D) \sum|v-u|<\eta
$$

where $F(u, v)=F(v)-F(u)$. Note that the Saks-Henstock Lemma [3, p. 11], for the $H_{1}$-integral holds. If, in addition, $D$ is $\delta_{2}$-fine partial division of $[a, b]$ with $\xi \in X_{2}$ such that

$$
\left|(D) \sum\{f(\xi)(v-u)-F(u, v)\}\right|<\epsilon,
$$

then $(D) \sum|v-u|<\eta$ implies $\left|(D) \sum f(\xi)(v-u)\right|<2 \epsilon$.

Now, take a $\delta$-fine division $D_{0}$ of $[a, b]$ such that $D_{0} \geq D_{1}, D_{2}$ using $\delta_{1}$ and a subset $E$ of $D_{0}$ covers $X_{1}$ with $\left|E-X_{1}\right|<\eta$. For any $\delta$-fine division $D \geq D_{0}$, take a $\delta_{2}$-fine division $D_{3}$ of those intervals in $D$ which are $\delta_{1}$-fine, namely, those with $\xi \in X_{1}$. Note that $\left(D_{3}\right) \sum f(\xi)(v-u)$ and $(D) \sum_{\xi \in X_{1}} f(\xi)(v-u)$ 
are sums over the same intervals, in which $D_{3}$ may contain $([u, v], \xi)$ with $\xi \in X_{1}$ and $\xi \in X_{2}-X_{1}$. Then we have

$$
\begin{aligned}
\mid(D) \sum_{\xi \in X_{2}} f(\xi) & (v-u)-A_{2}|\leq|(D) \sum_{\xi \in X_{1}} f(\xi)(v-u)-A_{1} \mid \\
& +\left|A_{1}-\left(D_{3}\right) \sum_{\xi \in X_{1}} f(\xi)(v-u)\right| \\
& +\left|\left(D_{3}\right) \sum_{\xi \in X_{2}} f(\xi)(v-u)+(D) \sum_{\xi \in X_{2}-X_{1}} f(\xi)(v-u)-A_{2}\right| \\
& +\left|\left(D_{3}\right) \sum_{\xi \in X_{2}-X_{1}} f(\xi)(v-u)\right| \\
< & 5 \epsilon .
\end{aligned}
$$

The proof is complete.

Theorem 5. Let $f$ be $H_{1}$-integrable on a closed set $X_{n}$ with primitive $F_{n}$ for $n=1,2, \ldots$, and $X=\cup_{n=1}^{\infty} X_{n}$. If $f$ is non-negative on $[a, b]$ and $F_{n}(b)-$ $F_{n}(a) \rightarrow A$ as $n \rightarrow \infty$, then $f$ is $H_{1}$-integrable on $X$.

Proof. We may assume that $X_{n} \subset X_{n+1}$ for each $n=1,2, \ldots$. Since $f$ is $H_{1}$-integrable on $X_{n}$, there exists $\delta_{n}(x)>0$ such that for any $\delta_{n}$-fine division $D$ of $[a, b]$ we have

$$
\text { (D) } \sum_{\xi \in X_{n}}\left|f(\xi)(v-u)-F_{n}(u, v)\right|<\frac{1}{2^{n}},
$$

where $F_{n}(u, v)=F_{n}(v)-F_{n}(u)$ and $F_{n}$ is the primitive of $f \mathcal{X}_{X_{n}}$ on $[a, b]$. Put $\delta(x)=\delta_{n}(x)$ when $x \in X_{n}-X_{n-1}$ with $X_{0}=\emptyset$, otherwise arbitrary. We may modify $\delta_{n}(x)$, if necessary, as in Lemma 4 . Since $f$ is non-negative on $[a, b]$, $f \mathcal{X}_{X_{n}}$ is absolutely $H_{1}$-integrable on $[a, b]$ and so $f \mathcal{X}_{X_{n}}$ is absolutely Henstock integrable on $[a, b]$. Thus, $F_{n}$ is absolutely continuous on $[a, b]$, and the result of Lemma 4 applies.

Given $\epsilon>0$, there exists an integer $N>0$ such that

$$
\left|A-F_{N}(a, b)\right|<\epsilon \quad \text { and } \quad \sum_{n=N+1}^{\infty} \frac{1}{2^{n}}<\epsilon .
$$

Further, there exists a $\delta$-fine division $D_{N}$ of $[a, b]$ such that for any $\delta$-fine $D \geq D_{N}$ of $[a, b]$ we have

$$
\left|(D) \sum_{\xi \in X_{N}}\left\{f(\xi)(v-u)-F_{N}(u, v)\right\}\right|<\epsilon .
$$


Here we have used Lemma 4 to obtain $D_{N}$ and the last inequality. Note that $F_{n}(u, v) \rightarrow F(u, v)$ as $n \rightarrow \infty$ and $0 \leq F(u, v)-F_{n}(u, v) \leq F(u, v)-F_{N}(u, v)$ for $n \geq N$. Here $F(a, b)=A$. Then for any $\delta$-fine division $D \geq D_{N}$ of $[a, b]$ we obtain

$$
\begin{aligned}
\left|(D) \sum_{\xi \in X} f(\xi)(v-u)-A\right| \leq & \left|(D) \sum_{\xi \in X_{N}}\left\{f(\xi)(v-u)-F_{N}(u, v)\right\}\right| \\
& +\sum_{n=N+1}^{\infty}\left|(D) \sum_{\xi \in X_{n}-X_{n-1}} f(\xi)(v-u)-F_{n}(u, v)\right| \\
& +\left|A-F_{N}(a, b)\right| \\
\leq & 3 \epsilon
\end{aligned}
$$

Hence $f$ is $H_{1}$-integrable on $X$.

With the idea presented in the proofs of Lemma 4 and Theorem 5, we can now look at the $H_{1}$-integrability of a Henstock integrable function. Note that the Cauchy Criterion [3, p. 10], also holds for $H_{1}$-integral. We give first the following lemmas.

Lemma 6. Let $X$ be a closed subset of $[a, b]$. If $f$ is $H_{1}$-integrable and bounded on $[a, b]$, then $f$ is $H_{1}$-integrable on $X$.

Proof. Let $|f(x)| \leq M$ for all $x \in[a, b]$. By the Cauchy Criterion, there exists $\delta(x)>0$ such that for each $\epsilon>0$ there is a $\delta$-fine division $D_{0}$ of $[a, b]$ such that for any $\delta$-fine divisions $D, D^{\prime} \geq D_{0}$ of $[a, b]$ we have

$$
\left|(D) \sum f(\xi)(v-u)-\left(D^{\prime}\right) \sum f(\xi)(v-u)\right|<\epsilon .
$$

Further, there exists a finite union $E$ of closed intervals such that $E \supset X$ and $|E-X|<\frac{\epsilon}{M}$. We can assume that a subset of $D_{0}$ forms a division of $E$. For every $\delta$-fine divisions $D_{1}, D_{2} \geq D_{0}$ of $[a, b]$, let $D_{1}^{*}$ and $D_{2}^{*}$ be the respective subsets of $D_{1}$ and $D_{2}$ which form divisions of $E$. Note that $\left(D_{i}\right) \sum_{\xi \in X} f(\xi)(v-u)=\left(D_{i}^{*}\right) \sum_{\xi \in X} f(\xi)(v-u)$ for $i=1,2$. We may assume further that

$$
\left(D_{1}\right) \sum_{\xi \in[a, b]-E} f(\xi)(v-u)=\left(D_{2}\right) \sum_{\xi \in[a, b]-E} f(\xi)(v-u) .
$$


Then

$$
\begin{aligned}
\mid\left(D_{1}\right) \sum_{\xi \in X} f(\xi)(v-u)- & \left(D_{2}\right) \sum_{\xi \in X} f(\xi)(v-u) \mid \\
\leq & \left|\left(D_{1}\right) \sum_{1} f(\xi)(v-u)-\left(D_{2}\right) \sum f(\xi)(v-u)\right| \\
& +\left(D_{1}^{*}\right) \sum_{\xi \notin X}|f(\xi)|(v-u)+\left(D_{2}^{*}\right) \sum_{\xi \notin X}|f(\xi)|(v-u) \\
< & 3 \epsilon .
\end{aligned}
$$

By the Cauchy Criterion again, the above inequalities imply that $f$ is $H_{1}$-integrable on $X$.

Lemma 7. Let $f$ be a measurable function on $[a, b]$. Then there exists a sequence $\left\{X_{i}\right\}$ of closed subsets of $[a, b]$ such that $f$ is $H_{1}$-integrable on each $X_{i}$ and $\left|[a, b]-\cup_{i=1}^{\infty} X_{i}\right|=0$.

Proof. It is well-known [2, p. 192] that there exists a sequence $\left\{\varphi_{n}\right\}$ of continuous functions on $[a, b]$ such that $\varphi_{n}(x) \rightarrow f(x)$ almost everywhere in $[a, b]$. By Egoroff's Theorem and Lemma 6 , for each $i=1,2, \ldots$, there is a closed set $X_{i} \subset[a, b]$ with $\left|[a, b]-X_{i}\right|<\frac{1}{i}$ such that $f$ is $H_{1}$-integrable on $X_{i}$. Obviously,

$$
\left|[a, b]-\cup_{i=1}^{\infty} X_{i}\right| \leq\left|[a, b]-\cup_{i=1}^{N} X_{i}\right| \leq\left|[a, b]-X_{N}\right|<\frac{1}{N} \rightarrow 0
$$

as $N \rightarrow \infty$, that completes the proof of the lemma.

Let a function $F$ be defined on $[a, b]$ and $X \subset[a, b]$. The function $F$ is said to be $A C^{*}(X)$ if for every $\epsilon>0$ there exists $\eta>0$ such that for any partial division $D=\{([u, v], \xi)\}$ of $[a, b]$ with $u$ or $v$ in $X$

$$
\text { (D) } \sum|v-u|<\eta \quad \text { implies } \quad(D) \sum|F(u, v)|<\epsilon .
$$

Further, $F$ is said to be $A C G^{*}$ on $[a, b]$ if $[a, b]$ is the union of $X_{1}, X_{2}, \ldots$ such that $F$ is $A C^{*}\left(X_{i}\right)$ for each $i$. This definition is equivalent to the definition in $[3$, p. 29] with $F$ being continuous on $[a, b]$ and to the classical definition in the book by Saks [4], that is, if $F$ is $A C G^{*}$ on $[a, b]$, then $F$ is continuous there. It was shown in [3, p. 34] that is $f$ is a Henstock integrable function on $[a, b]$, then its primitive $F$ is $A C G^{*}$ on $[a, b]$ and we can assume that $[a, b]=\cup X_{i}$ such that $F$ is $A C^{*}\left(X_{i}\right)$ and $X_{i}$ is closed for each $i$.

On the other hand, a sequence $\left\{F_{n}\right\}$ of functions defined on $[a, b]$ is said to be $U A C^{*}(X)$ where $X \subset[a, b]$ if, in the definition of $A C^{*}(X), \eta>0$ is 
independent of $n$. Further, $\left\{F_{n}\right\}$ is $U A C G^{*}$ on $[a, b]$ if $[a, b]=\cup X_{i}$ such that $\left\{F_{n}\right\}$ is $U A C^{*}\left(X_{i}\right)$ for each $i$ and we can assume that $X_{i}$ is closed for each $i$. Futhermore, the sequence $\left\{F_{n}\right\}$ is said to be oscillation-convergent to some function $F$ defined on $[a, b]$ if $[a, b]=\cup X_{i}$ with $X_{i}$ being closed and for every $i$ and $\epsilon>0$ there is an integer $N>0$ such that for any partial division $D=\{([u, v], \xi)\}$ of $[a, b]$ with $\xi$ in $X_{i}$ we have

$$
\sum\left|F_{n}(u, v)-F(u, v)\right|<\epsilon
$$

whenever $n \geq N$. The following lemma was proved in [3, p. 56]

Lemma 8. Let $\left\{f_{n}\right\}$ be a sequence of Henstock integrable functions on $[a, b]$ and is control-convergent to some function $f$ on $[a, b]$; that is, the following conditions are satisfied:

1. $f_{n}(x) \rightarrow f(x)$ almost everywhere in $[a, b]$ as $n \rightarrow \infty$;

2. the sequence $\left\{F_{n}\right\}$ of primitives of $\left\{f_{n}\right\}$ is $U A C G^{*}$ on $[a, b]$; and

3. $\left\{F_{n}\right\}$ converges uniformly on $[a, b]$.

Then $\left\{F_{n}\right\}$ is oscillation-convergent to the primitive $F$ of $f$ on $[a, b]$.

Lemma 9. Let $f$ be Henstock integrable on $[a, b]$ with primitive $F$. Then there exists a sequence $\left\{F_{n}\right\}$ of absolutely continuous functions that is oscillationconvergent to $F$ on $[a, b]$.

Proof. Since $f$ is Henstock integrable on $[a, b], F$ is $A C G^{*}$ on $[a, b]$; that is, there exists a sequence $\left\{X_{n}\right\}$ of closed subsets of $[a, b]$ such that $F$ is $A C^{*}\left(X_{n}\right)$ for each $n$. We may assume that $X_{n} \subset X_{n+1}$ and $a, b \in X_{n}$ for each $n$. Since $X_{n}$ is closed, we can write $(a, b)-X_{n}=\cup_{k=1}^{\infty}\left(a_{k}, b_{k}\right)$ and put

$$
F_{n}(x)= \begin{cases}F(x) & \text { when } x \in X_{n} ; \\ F\left(a_{k}\right)+\frac{F\left(b_{k}\right)-F\left(a_{k}\right)}{b_{k}-a_{k}}\left(x-a_{k}\right) & \text { when } x \in\left(a_{k}, b_{k}\right) \text { for all } k .\end{cases}
$$

Since $f$ is Henstock integrable on $[a, b]$, its primitive $F$ and thus $F_{n}$ are continuous on $[a, b]$. Further, we can assume that $F_{n} \rightarrow F$ uniformly as $n \rightarrow \infty$.

Since $F_{n}$ is $A C^{*}\left(X_{n}\right)$ for each $n$, given an $\epsilon>0$ there exists $\eta_{n}>0$ such that for any partial division $\pi_{n}$ of $[a, b]$ with $u$ or $v$ in $X_{n}$

$$
\left(\pi_{n}\right) \sum|v-u|<\eta_{n} \quad \text { implies } \quad\left(\pi_{n}\right) \sum\left|F_{n}(u, v)\right|<\epsilon .
$$

Let $N$ be fixed. For $n \geq N, F_{n}(x)=F_{N}(x)$ for all $x \in X_{N}$. Thus we can choose $\eta=\min _{1 \leq i \leq N}\left\{\eta_{i}\right\}$ for all $F_{n}$ so that $\left\{F_{n}\right\}$ is $U A C^{*}\left(X_{N}\right)$. Hence $\left\{F_{n}\right\}$ is $U A C G^{*}$ on $[a, b]$. 
Now define

$$
f_{n}(x)= \begin{cases}f(x) & \text { when } x \in X_{n} \\ \frac{F\left(b_{k}\right)-F\left(a_{k}\right)}{b_{k}-a_{k}} & \text { when } x \in\left(a_{k}, b_{k}\right) \text { for all } k .\end{cases}
$$

It is easy to see that $f_{n}$ is Henstock integrable on $[a, b]$ and $F_{n}$ is the primitive of $f_{n}$ for each $n$. Since $X_{n} \uparrow[a, b], f_{n}(x) \rightarrow f(x)$ almost everywhere in $[a, b]$.

From the above discussion, $\left\{f_{n}\right\}$ is control-convergent to $f$ on $[a, b]$. Thus, by Lemma $8,\left\{F_{n}\right\}$ is oscillation-convergent to $F$ on $[a, b]$. The proof is complete.

We now give the main result of the paper.

Theorem 10. Let $f$ be Henstock integrable on $[a, b]$. Then there is an $H_{1}$-integrable function $g$ such that $f(x)=g(x)$ almost everywhere in $[a, b]$.

Proof. By Lemma 7, there exists a sequence $\left\{X_{i}\right\}$ of closed subsets of $[a, b]$ such that $f$ is $H_{1}$-integrable on each $X_{i}$ and $\left|[a, b]-\cup_{i=1}^{\infty} X_{i}\right|=0$. Let $X=$ $\cup_{i=1}^{\infty} X_{i}$ and $X_{i} \subset X_{i+1}$ for each $i$. We prove that $f$ is $H_{1}$-integrable on $X$.

We may assume that the result of Lemma 9 holds; that is, for every $i$ there exists an integer $n(i) \geq i$ such that for any partial division $D$ of $[a, b]$ with $u$ or $v$ in $X_{i}$, we have

$$
\text { (D) } \sum\left|F_{n(i)}(u, v)-F(u, v)\right|<\frac{1}{2^{i}},
$$

where $F$ is the primitive of $f$ on $[a, b]$ and $F_{n(i)}(x)$ is as defined in the proof of Lemma 9.

Since $f$ is $H_{1}$-integrable on $X_{n(i)}$ for each $i, f_{n(i)}$ is also $H_{1}$-integrable on $X_{n(i)}$, where $f_{n(i)}$ is as defined in Lemma 9. There exists $\delta_{n(i)}(x)>0$ such that for any $\delta_{n(i)}$-fine division $D$ of $[a, b]$ we have

$$
\text { (D) } \sum_{\xi \in X_{i}}\left|f_{n(i)}(\xi)(v-u)-F_{n(i)}(u, v)\right|<\frac{1}{2^{i}} .
$$

For $i=1,2, \ldots$, put $\delta(x)=\delta_{i}(x)$ if $x \in X_{i}-X_{i-1}$ with $X_{0}=\emptyset$; otherwise, put $\delta(x)>0$ arbitrary. We may modify $\delta_{i}(x)$, if necessary, as in Lemma 4.

Given $\epsilon>0$, there exists a positive integer $N=n\left(i_{0}\right)$ such that

$$
\sum_{i=i_{0}+1}^{\infty} \frac{1}{2^{i}}<\epsilon .
$$


Further, there exists a $\delta$-fine division $D_{N}$ of $[a, b]$ such that for any $\delta$-fine division $D \geq D_{N}$ of $[a, b]$ we have

$$
\left|(D) \sum_{\xi \in X_{i_{0}}}\left\{f(\xi)(v-u)-F_{N}(u, v)\right\}\right|<\epsilon .
$$

For any $\delta$-fine division $D=\{([u, v], \xi)\}$ of $[a, b]$ with $\xi \in X_{n}-X_{n-1}$ and $D \geq D_{N}$, we have

$$
\begin{aligned}
\left|(D) \sum_{\xi \in X}\{f(\xi)(v-u)-F(u, v)\}\right| \leq\left|(D) \sum_{\xi \in X_{i_{0}}}\left\{f(\xi)(v-u)-F_{N}(u, v)\right\}\right| \\
+\sum_{i=i_{0}+1}^{\infty}\left|(D) \sum_{\xi \in X_{i}-X_{i-1}}\left\{f(\xi)(v-u)-F_{n(i)}(u, v)\right\}\right| \\
+\left|(D) \sum_{\xi \in X_{i_{0}}}\left\{F_{N}(u, v)-F(u, v)\right\}\right| \\
+\sum_{i=i_{0}+1}^{\infty}\left|(D) \sum_{\xi \in X_{i}-X_{i-1}}\left\{F_{n(i)}(u, v)-F(u, v)\right\}\right| \\
<\epsilon+\sum_{i=i_{0}+1}^{\infty} \frac{1}{2^{i}}+\frac{1}{2^{i_{0}}}+\sum_{i=i_{0}+1}^{\infty} \frac{1}{2^{i}} \\
<4 \epsilon .
\end{aligned}
$$

Therefore, $f$ is $H_{1}$-integrable on $X$.

Corollary 11. A function $f$ is Henstock integrable on $[a, b]$ if and only if $f(x)=g(x)$ almost everywhere in $[a, b]$ for some $H_{1}$-integrable function $g$ on $[a, b]$.

It is not known whether every Henstock integrable function on $[a, b]$ is also $H_{1}$-integrable there. We conjecture that it is not.

\section{References}

[1] N. Dunford and J. T. Schwartz, Linear Operators I, Interscience 1958.

[2] C. Goffman, Real Functions, Rinehart 1953.

[3] P. Y. Lee, Lanzhou Lectures in Henstock Integration, World Scientific 1989.

[4] S. Saks, Theory of the Integral, 2nd ed, Hafner 1937. 
